\title{
Subject Index Vol. 42, 1996
}

Supplement Issue No. 1 has its own Subject Index

Aerobic exercise 204 Age-related changes 290

-, rat 229

maculopathy 257 Aged 46,199

Aging 7, 18,25,69,87, 137,147,211,218,235,322

Aldosterone 229

Alzheimer's disease 170

Amantadine, influenza therapy 280

Anaerobes 40

Anti-CD3 137

Asthma, elderly patients 61

-, potentially fatal 183

Atrial natriuretic factor, age-related changes 79

Bacterial overgrowth 40 Bed rise difficulty 294 Body composition 330

- $\quad$ sway 199

Brain 87

Catalase 312

Cataract 257

CD4+/CD45RA+ 69

CD4+/CD45RO+ 69

Cerebellum 170

Chronic obstructive airways disease 61

Cognitive disorders 199

Collagen 218

Confusion 104

Corticosteroids 183

Dehydration 36 Delirium 36 Dementia 36,241 Diaphragm 218 Disc degeneration 322

Docosahexaenoic acid 25 Down's syndrome 147 Drosophila aging 123

- melanogaster 14,235

Drugs utilization 199

Echocardiography 54 EF-l $\alpha 123$ Eicosapentaenoic acid 25

Elastic fibers 211

Elderly 1,40,163,330,339,348

Chinese, tuberculosis 155

high-risk influenza patients 280 -, influenza vaccination 270

people 190

women 204 Estradiol benzoate 18 Extracellular matrix 218

Falls 108

-, accidental 199 
-, elderly 104

-, previous history 104

-, risk factors 104

Fasting hydrogen level 40

Fischer 344 rats 25

Fish oil 25

Functional ability, assessment 294

Gait 199

analysis 7

prediction, stroke 348 Gastric atrophy 97

blood flow 290 Genetics of aging 14 Gut brain peptides 241

Helicobacter pylori 97

Hematology 301

High-risk patients 190

Hippocampus 170

Human heart 211

Hydrogen breath test 40

Hypergravity 235

Hypertensive intracerebral hematomas 339

Hypertonic volume expansion 79

Hypothalamic peptides 241

Immune response 190

- $\quad$ system 87

Incontinence, diarrhoea 104 Influenza 274

-, morbidity, elderly 270

vaccination 190

-, elderly 270

Inhibitor factor kappa B 137 Inpatients, geriatric 36 Insulin-like growth factor-I 330 Interleukin269 Intervertebral disc 322 Intestinal metaplasia 97 Intracerebral hemorrhage 339 Intrinsic asthma 183

Learning 301

Left-ventricular outflow tract obstruction 54

Life span 14, 312

Lipid peroxidation 312

Lymphocyte subsets 69

Management 61

Mantoux test 155

Maximal strength training 114

Mechanics indices 46

Melatonin, side effects 87

Memory cells 69

Metered dose inhalers 183

Mitral valve ring calcification 54

Morbidity 301

Morphine 79 
Morphometry 170,211

mRNA 123

Muscle fiber type 218

- strength 199

Muscular endurance 204

Naive cells 69

Neuron number 18

Neuronal metabolism 170

Neuropeptides 241

Neurosurgery 1

Nonsteroidal anti-inflammatory drugs 290

Nuclear factor kappa B 137

Nucleolus organizer regions 147

Ocular ageing $257 \omega-3$ fatty acids 25 Operative results 339

Peak torque 114 Peroxidase 312 Phorbol myristate acetate 137 Physiology 199 Pituitary peptides

241 Plasma renin activity 229 Posture 46 Presbyopia 257

Principal lifetime occupation 163 Proboscis extension response 235 Prophylaxis, influenza 280

Proprioception 199 Protein synthesis components 123 Proximal septal hypertrophy 54

Radical scavenger 87 Rat(s) 7

- $\quad$ aorta 25

Reaction time 199

Rectus abdominis 218

Reproduction at late age 14

Residential homes, tuberculosis 155

Ribosomal genes 147

Rimantadine, influenza therapy 280

Rodent 301

rRNA 123

SDAT 170 SDN-POA 18

Senile dementia, Alzheimer type 108

Serine proteinase inhibitor 322

Sitting equilibrium 348

Skeletal muscle 218

Sleep disturbances 163

Social factors 163

Sodium deprivation 229

Soluble interleukin-2 receptor 69

Stomach 290

Stride length variability 108

Stroke 348

Subcutaneous infusions 36

Synaptic plasticity 170

Total body bone mineral content 330

- $\quad$ - lean mass 330

Training 204

Transcriptional activity 147 Treadmill running 7 tRNA 123 
Tumor necrosis factor-alpha 137

Ultrastructure 211 Urinary creatinine 114

Vaccination 274 Vascular smooth muscle 25 Vision 199 Vitamin E 312

Zaprionus paravittiger 312

356

Subject Index Vol. 42, 1996 